\section{P-107 IMPROVING THE EXPERIENCE OF PEOPLE WITH ADVANCED DEMENTIA AT THE END OF LIFE IN GREENWICH AND BEXLEY BOROUGHS}

Alison McCarthy, Lisa Morris. Greenwich and Bexley Community Hospice, London, UK

10.1136/bmispcare-2017-hospice.133

Background It is recognised that people with dementia have been poorly served by hospices and palliative care services, leading to lack of support, inappropriate hospital admissions and poor quality care at end of life. Previously our hospice supported only 50 people a year with dementia and our staff had little understanding or experience of their specific needs.

Aims The aim of the project was to enable more people with dementia to access quality specialist palliative care in the place of their choosing, improving their care at end of life.

Method With the support of a St James's Place Foundation grant, we employed one of our Clinical Nurse Specialists 2.5 days a week to focus on advanced dementia. In addition to managing a caseload of patients, she provided expert training, advice and support across the hospice. Facilitating improvements in the way we identified and supported people with dementia and ensuring they received appropriate care within our own and other services. We worked in close collaboration with local community Advanced Dementia Service, cross-referring patients and joint visiting. This enabled patients to have the most timely support and referral into all appropriate palliative care services.

Results Patients with dementia, supported by the hospice increased by over $200 \%$. They were able to access all palliative care services and feedback was excellent. Hospital admissions were prevented and $94 \%$ of patients that died did so in their preferred place of death. Over 200 hospice and local community staff received education on dementia.

Conclusion Following the project, people with advanced dementia and their families have had greater access to palliative care services. More people have had support to enable them to stay where they wanted to die. The knowledge, skills and therefore confidence of staff and volunteers has increased, which supports better care. Dementia is firmly on our hospice agenda.

\section{P-108 COMMUNICATING DO NOT ATTEMPT CARDIOPULMONARY RESUSCITATION DECISIONS WITH PATIENTS AND FAMILIES}

Jenny Hadley. Compton Hospice, Wolverhampton, UK

10.1136/bmjspcare-2017-hospice.134

Background Current policy emphasises the importance of including palliative and end of life patients and their families in decisions about their care (House of Commons Health Committee, 2015; National Institute for Health and Care Excellence, 2017) with clear, sensitive and timely communication (Leadership Alliance for the Care of Dying People, 2014; National Palliative and End of Life Care Partnership, 2015). Do Not Attempt Cardiopulmonary Resuscitation (DNACPR) conversations can help patients accept their predicament (Low et al., 2014) and may help to achieve a dignified death (Mack et al., 2012). However, there is little research detailing how these conversations take place or the terminology and approaches used.

Aims This research study explored the experiences of Clinical Nurse Specialists (CNSs) in palliative care when managing Do Not Attempt Cardiopulmonary Resuscitation (DNACPR) conversations with patients and their families.

Methodology Six semi-structured interviews were conducted in a hospice setting. The results were presented using an autoethnographic approach, exploring the author's experiences in addition to those of colleagues (CNSs) selected by purposive sampling. Data was analysed using NVivo11 applying thematic analysis.

Results Four main themes emerged; learning from experiences, feeling responsible to get it right, how conversations are initiated and the barriers to DNACPR discussions. DNACPR conversations are particularly difficult where patients and families are informed inappropriately or insensitively about DNACPR and when there is little rapport established. CNSs are challenged in balancing sensitivity whilst also communicating clearly about key decisions that are essential to supporting a dignified death.

Conclusion Fears associated with communicating DNACPR decisions may lead to patients receiving information inappropriately, insensitively and without clarity. Raising awareness of these research findings amongst healthcare professionals can lead to enhanced communication provided in a clear, timely and sensitive manner by a healthcare professional with whom patients have an existing rapport.

\section{P-109 HOSPICE NURSES' THOUGHTS AND FEELINGS OF 'DNACPR' DISCUSSION WITH PATIENTS}

Alison Pilsworth, Christina Faull, Zoebia Islam. LOROS Hospice, Leicester, UK

\subsection{6/bmjspcare-2017-hospice. 135}

Aims This study explored the thoughts and feelings of hospice nurses regarding Do Not Attempt Cardiopulmonary Resuscitation (DNACPR) discussions and the potential extension of their role in this including formal documentation of the outcome of that discussion by signing the DNACPR form.

Methods Eight semi-structured interviews were carried out with senior hospice inpatient and day therapy nurses. Interpretive phenomenological analysis was used to explore the shared lived meaning of this phenomenon.

Results Four themes emerged:

1. Current practice around hospice nurses

2. How proposed changes to practice may enhance patient experience

3. The benefits to hospice nurses

4. The challenges of this change in role.

Participants appeared confident with the concept of nurses discussing and documenting DNACPR decisions. They could envisage that seamless advance care planning would enhance patient care, and that nurses' rapport with patients made them 